\section{Engines of change}

John Forty

Supercomputers and Their Use. By Christopher Lazou. Clarendon: 1986. Pp.227. £22.50, \$45.

THE generally accepted definition of a supercomputer is a general purpose computer which is the most powerful computing engine available at any given time. In principle, therefore, there can only be one such machine but in practice a range of supercomputers is available and the choice depends on performance for the particular application. Inevitably the pace of advance in computer technology is such that no machine can hold the title for more than a few years; thus, in its day, the Ferranti Atlas was a supercomputer but was soon overtaken by the very powerful mainframes of the 1960 s such as the IBM $360 / 195$ and the CDC 7600 .

In the past decade there have been enormous advances in computing power, defined loosely in terms of the ability to deal with the very large scale computation needed to solve complex problems, usually of a scientific nature. These have come about largely as a result of the development of new forms of computer architecture. Currently the most powerful

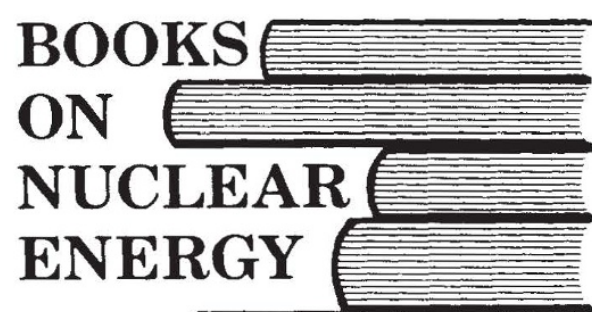

for the specialist for the public

\section{For our free catalog call or write:}

AMERICAN NUCLEAR SOCIETY

555 N. Kensington Avenue

La Grange Park, Illinois 60525 312-352-6611

Reader Service No.7 machines commercially available are the Cray and Control Data Corporation ranges, which exploit the concepts of multiple central processing and architectures which permit pipelining of a series of similar processing steps which can thereby be processed simultaneously, and consequently increase the speed of calculation. The latest Crays incorporate up to four powerful central processors so that this vector processing of data can be performed in parallel. Machines such as the ICL DAP (distributed array processor), consisting of a very large number of simple processing units working in parallel, are extremely effective in carrying out large scale computations where there is a large number of similar tasks to be performed simultaneously. Computing engines of this kind are orders of magnitude more powerful than the conventional mainframe.

A useful measure of progress over the past 20 years can be obtained by comparing peak performances, today's multiprocessor machines, such as the 4processor Cray X-MP48, being several thousand times faster than the Atlas. Advances in hardware technology have also resulted in a reduction of the cost per computational step by a similar factor. The result is an improvement in performance which is the equivalent of travelling at ten times the speed of Concorde compared with walking pace, for the same cost!

Computer design and engineering is continually evolving, and it might be thought that any account of supercomputers as we know them today could be of little permanent value. That may be so, but Christopher Lazou's little book does serve a useful purpose. It draws attention to the emerging scientific method of computation which is now seen by scientists to be a valid and technically feasible approach to solving problems (usually but not always of a scientific nature) which are so complex that the traditional approaches of experiment or analytical investigation are impractical. Supercomputers have made it possible to study the behaviour of the Earth's atmosphere and oceans in great detail, which will lead to a better understanding of climate, weather and so on. We can produce computer models of whole oil reservoirs to optimize output. And aircraft designers are able to design parts of, or even whole aeroplanes, and simulate the aerodynamic performance over a wide range of flight conditions with such realism that the time-consuming and expensive process of wind-tunnel modelling is no longer necessary. An important aim of the author has been to draw attention to the strategic importance of the supercomputer to the many areas of scientific research and engineering design in which it is now being applied.

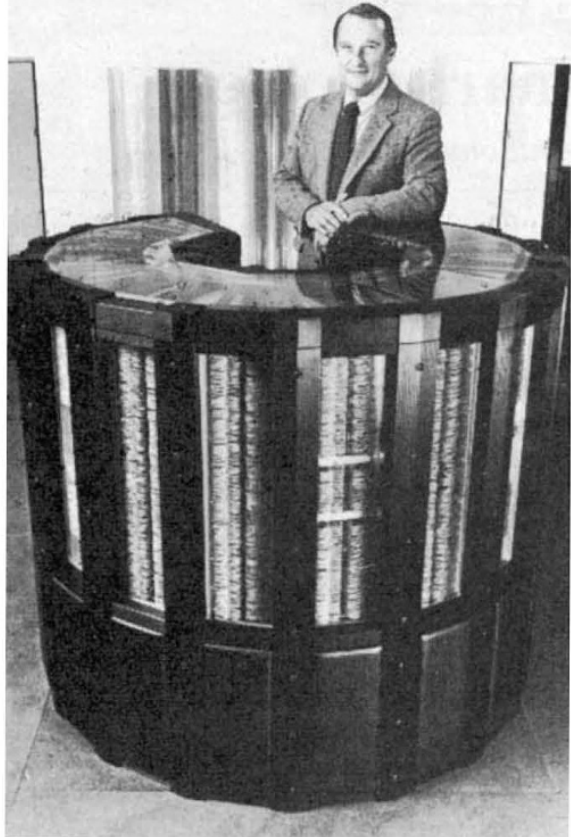

Cray and his creation - Seymour Cray with the Cray-2 machine, announced in June 1985.

The basic concepts of supercomputers, the principles underlying their architecture and the developments in semiconductor technology which, of course, are fundamental to their performance, are set out in the first four chapters. The treatment is inevitably superficial, but the text and well-chosen illustrations make it easy to grasp the essential features. There are also some useful chapters on scientific computational methods, including scalar and vector techniques, and optimal vectorization of programs. The reader is introduced to the concept of performance in terms of hardware and software parameters such as the number of parallel processors, degree of vectorization and the fraction of work that can be processed in parallel. As its title declares, the book also covers the uses of supercomputers and a number of examples are described, drawn largely from the author's experience with the Cray 1 (the supercomputer of the late 1970s) at the University of London Computer Centre. Looking forward, there is a useful survey at the end of the book of new supercomputers known to be in the pipeline and of future developments that should be made possible by advances in technology.

Lazou writes in a fluent, informative manner. Although the subject is not explored in depth, the book provides a good introduction for those who are already computer literate and wish to know more about these developments at the forefront of computer technology.

John Forty is Vice-Chancellor and Principal of the University of Stirling, Stirling FK9 4LA, $U K$. He is the author of Future Facilities for Advanced Research Computing, the report of $a$ Joint Working Party of the Advisory Board for the Research Councils, the Computer Board and the University Grants Committee. 